\title{
Vordere Schulterluxation reponieren - Schritt für Schritt
}

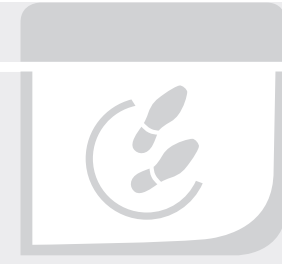

Peter Melcher, Jan Theopold

Je schneller eine luxierte Schulter reponiert werden kann, desto geringer ist das

Risiko von Nervenverletzungen und Folgeschäden. Doch bedeutet dies zwingend

eine präklinische Reposition?

\section{ABKÜRZUNG}

pDMS periphere Durchblutung, Motorik und Sensibilität

\section{Grundlagen}

Das Schultergelenk ist bei Luxationen das am häufigsten betroffene Gelenk. Die Hauptursachen sind meist ein adäquates Trauma oder eine chronische Instabilität. Vor allem Wurf- oder Vollkontaktsportler zeigen ein erhöhtes Risiko für diese Verletzung, aber auch Snowboarder und Surfer sind nicht selten betroffen.

Mehr als 95\% der Schulterluxationen erfolgen nach ventral [1]. Junge erwachsene Männer erleiden diese Verletzung am häufigsten nach einem adäquaten Trauma im Sinne eines Gegnerkontakts bei abduziertem, außenrotiertem Arm ( $\vee$ Abb. $\mathbf{1}$ ) oder im Rahmen eines Sturzes auf den ausgestreckten Arm.

Ein Sturz nach vorne, auf den ausgestreckten Arm, kann auch eine - mit 3\% aller Schulterluxationen deutlich seltenere - posteriore Luxation ( $\bullet$ Abb. 2 ) auslösen. Auch wenn die posteriore Schulterluxation sehr selten ist, sollte sie nicht übersehen werden. Eine Chronifizierung bedeutet in den meisten Fällen eine operative Therapie bis hin zur endoprothetischen Versorgung. Als Raritäten sind die superioren Luxationen und die Luxatio erecta ( Abb. 3) zu finden.

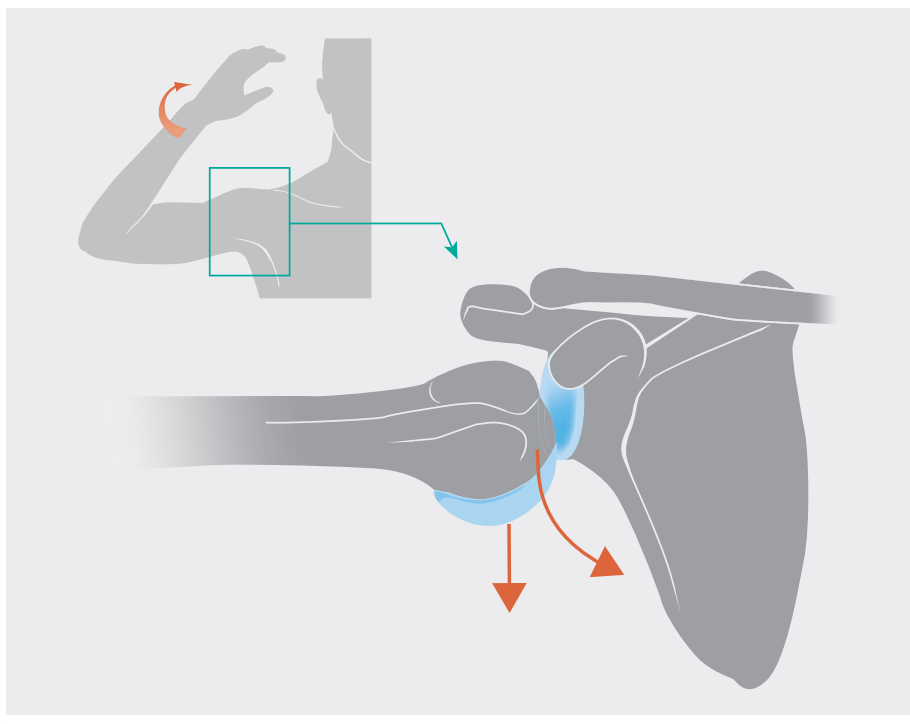

- Abb. 1 Durch Gegnerkontakt an Unterarm und Hand, beispielsweise in der Wurfbewegung, kann eine vordere Schulterluxation ausgelöst werden.

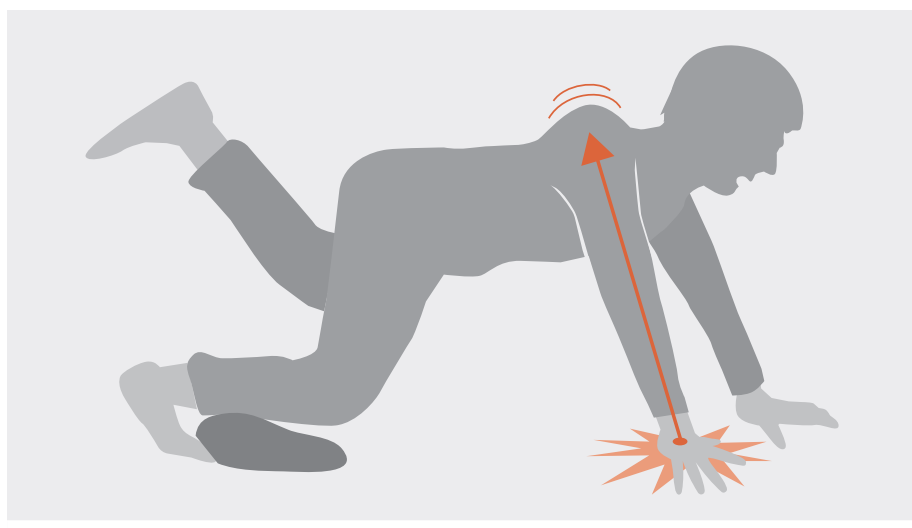

- Abb. 2 Durch einen Vorwärtssturz auf den ausgestreckten Arm kann eine hintere Schulterluxation provoziert werden. Analog kann ein Rückwärtssturz auf die ausgestreckten Arme eine vordere Schulterluxation auslösen. 


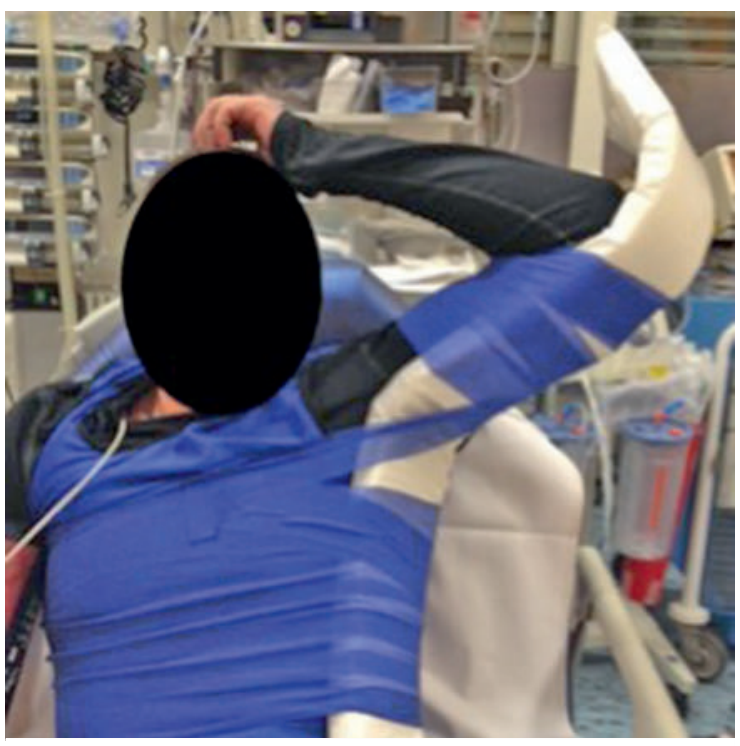

- Abb. 3 Klinisches Bild einer Luxatio erecta. Der Patient kann durch die verhakte Situation den Arm nicht mehr adduzieren.

\section{Wann Schulter reponieren - und wann nicht?}

\section{Präklinische Diagnostik}

Zur Erstdiagnostik sollte die Schulter inspiziert und im Anschluss eine Bewegungsprüfung durchgeführt werden. Auffällig wird die Schulterluxation meist durch eine schmerzhafte Bewegungseinschränkung. Die Gelenkkonturen sind im Seitenvergleich verändert und ggf. das lee-

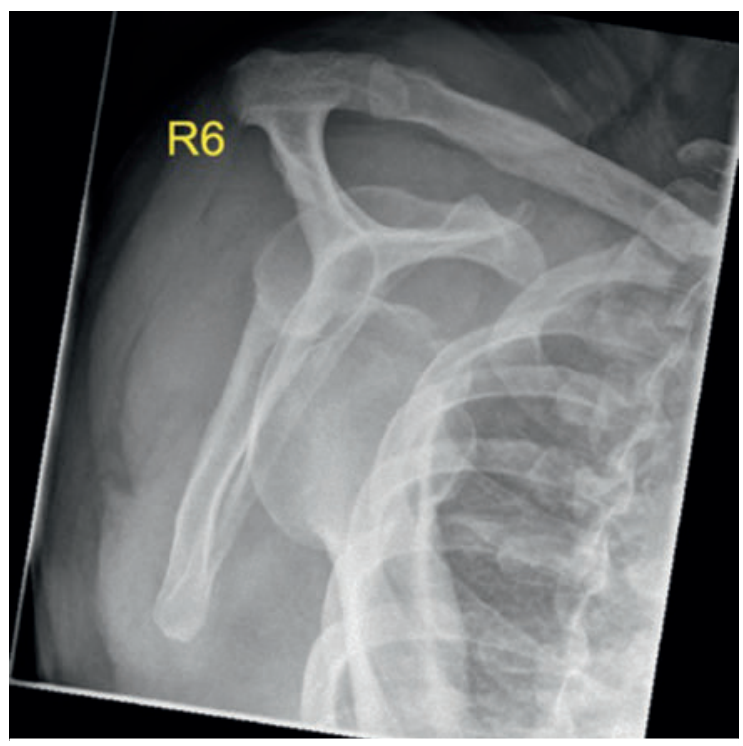

- Abb. 4 Röntgenbild einer rechten Schulter in Y-Aufnahmetechnik mit Nachweis einer vorderen Schulterluxation. re Glenoid palpabel. Bei offensichtlicher Fehlstellung und eingeschränkter Beweglichkeit besteht somit der Verdacht auf eine Luxation. Es sollten orientierend periphere Durchblutung, Motorik und Sensibilität (pDMS) der Extremität geprüft werden. [3]

\section{TAKE HOME MESSAGE}

Nur bei auffälliger pDMS (periphere Durchblutung, Motorik und Sensibilität) besteht die Indikation zur sofortigen Reposition. In allen anderen Fällen ist ein zeitnaher Transport unter ausreichender Analgesie in die nächste Klinik anzustreben.

\section{Klinische Diagnostik}

Innerklinisch wird erneut die oben genannte klinische Diagnostik durchgeführt und durch Röntgen im anterior-posterioren und axialen oder Y-Strahlengang ergänzt ( Abb. 4, Abb. 5).

\section{Merke}

Die Röntgenbildgebung dient zur Sicherung der Diagnose bzw. dem Ausschluss einer Fraktur oder von Begleitverletzungen und ist somit nicht zuletzt aus forensischen Gründen einer präklinischen Reposition vorzuziehen. Sie ist zudem wichtig, da $60 \%$ der dorsalen Schulterluxationen bei initialem Arztkontakt übersehen werden und zu einer chronischen Luxation führen können [4].

Die schonende Reposition erfolgt nach der Diagnosesicherung.

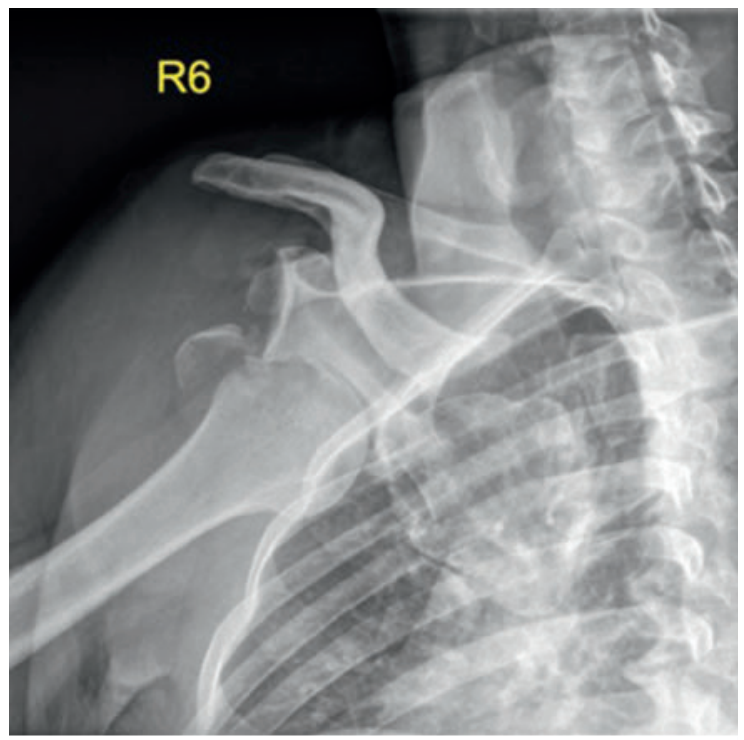

- Abb. 5 Röntgenbild einer rechten Schulter in a.-p. Strahlengang mit Nachweis einer Schulterluxation mit Tuberculum-majus-Fraktur und Glenoidfraktur. 


\section{TAKE HOME MESSAGE}

Vor der Reposition sollte, abgesehen von wenigen Ausnahmefällen, immer eine Röntgendiagnostik erfolgen.

\section{Technik der Schulterreposition}

Eine Vielzahl von Repositionstechniken ist beschrieben. Während Patienten mit Erstluxationen häufig in Analgosedierung reponiert werden müssen, können Patienten mit rezidivierenden Luxationen auch zur Selbstreposition, z. B. nach Stimson, angeleitet werden.

TIPP

Die Reposition lässt sich durch adäquate Analgesie und ggf. auch milde Sedierung zur Muskelentspannung deutlich erleichtern.

\section{Cave}

Harsche oder ruckartige Repositionsversuche sind unbedingt zu vermeiden, um Folgeverletzungen wie Plexusläsionen, Weichteilschäden oder Frakturen zu verhindern.

\section{Schulterreposition nach Matsen}

\section{Schritt 1 Hilfsmittel anlegen}

Sie können die Reposition allein oder mit Helfer durchführen. In jedem Fall legen Sie sich ein Hilfsmittel (z. B. ein Tuch) ringförmig um das Becken und verknoten es ( Abb. 6).

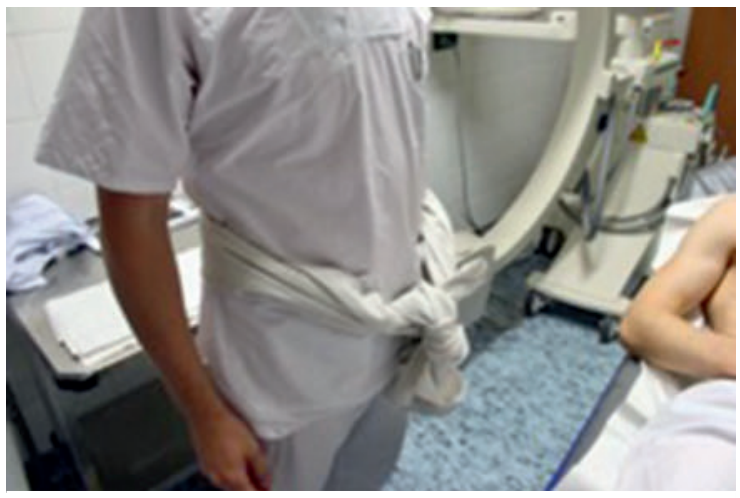

Abb. 6 Knoten Sie sich vor der Schulterreposition ein Hilfsmittel (z. B. eine Unterlage) um die Taille.

\section{Schritt 1a Helfermethode}

Bei zu erwartender erschwerter Reposition sollten Sie einen Helfer hinzuziehen und ein Tuch zum Gegenhalten einmal um den Thorax des Patienten legen ( $\bullet$ Abb. 7).

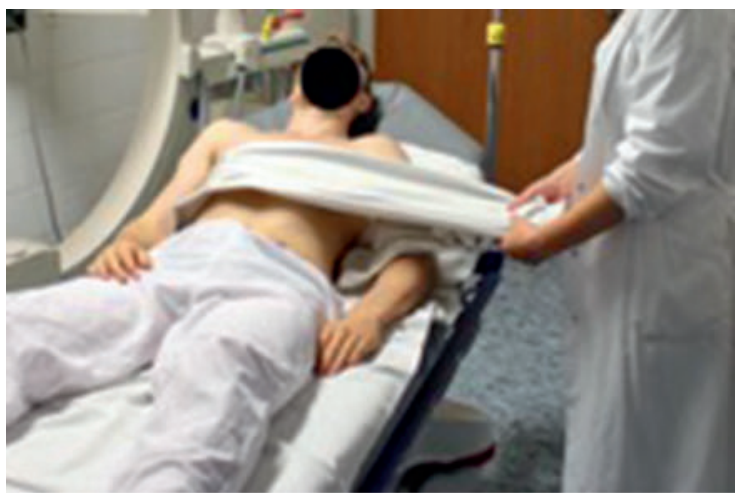

- Abb. 7 Legen Sie eine weitere Unterlage für die Helfermethode um den Brustkorb des Patienten für einen Gegenhalt.

\section{Schritt 2 Arm des Patienten positionieren}

Beim ausreichend analgosedierten Patienten führen Sie den Unterarm durch den umgelegten Gurt und flektieren im Ellenbogen um $90^{\circ}$. Zusätzlich halten Sie den Arm leicht abduziert ( $\triangleright$ Abb. 8). Übliche Dosierungen für häufig zur Schulterreposition verwendete Medikamente sind in > Tab. 1 aufgeführt.

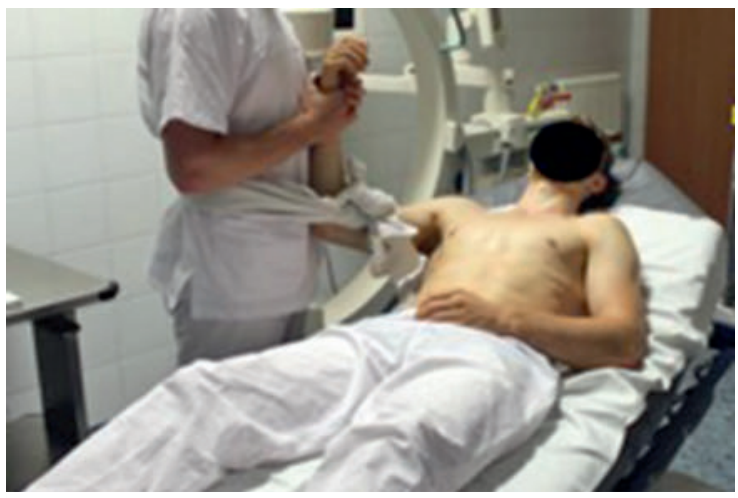

- Abb. 8 Legen Sie für die Reposition den gebeugten Arm des Patienten in die Schlaufe und greifen Sie zusätzlich das Handgelenk. 
- Tab. 1 Dosierungsvorschläge für gebräuchliche Medikamente bei einer Schulterreposition.

\begin{tabular}{|l|l|l|}
\hline Präparat & Dosis & Richtdosis $\mathbf{7 0 - 8 0 ~} \mathbf{k g K G}$ \\
\hline Fentanyl & $0,6-2,5 \mu \mathrm{g} / \mathrm{kgKG}$ i.v. & $0,05-0,2 \mathrm{mg}$ i.v. \\
\hline Propofol & $0,5-1 \mathrm{mg} / \mathrm{kgKG}$ i.v. & $60 \mathrm{mg} \mathrm{i.v.}$ \\
\hline Midazolam & $0,03-0,1 \mathrm{mg} / \mathrm{kgKG}$ i.v. & $1-2 \mathrm{mg}$ i.v. \\
\hline Esketamin & $0,3-0,5 \mathrm{mg} / \mathrm{kgKG}$ i.v. & $25-50 \mathrm{mg}$ i.v. \\
\hline
\end{tabular}

\section{Schritt 3 Zug ausüben}

Durch langsames Verlagern des Körpergewichtes können Sie stetigen Zug auf den Arm ausüben, bis die Schulter reponiert ist ( $\triangleright$ Abb. 9).

TIPP

Die zusätzliche Außenrotation des Patientenarmes kann auch bei ausbleibender Reposition noch zum Zurückspringen des Kopfes in die Pfanne führen.

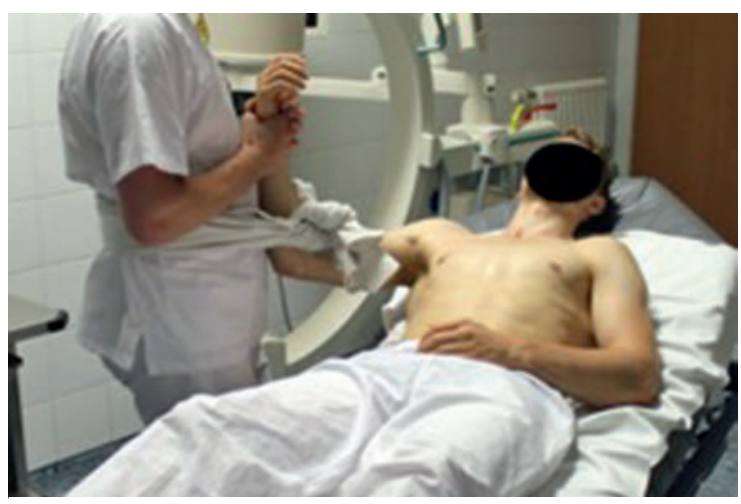

- Abb. 9 Verlagern Sie Ihr Körpergewicht langsam nach hinten, um gleichmäßigen, stetigen Zug auf die Schulter auszuüben, bis die Schulterreposition erfolgt ist.

\section{Schritt 3 a Helfermethode}

Durch den Einsatz eines Helfers kann ein Gegenzug aufgebaut werden. Hierbei ist darauf zu achten, keine übermäßige Gewalt einwirken zu lassen ( $\triangleright$ Abb. 10$)$. Sollte die Schulter nicht zu reponieren sein, ist ggf. die Analgosedierung zu vertiefen. Auch eine Außenrotation der luxierten Schulter kann versucht werden. In seltenen Fällen liegt ein Repositionshindernis vor.

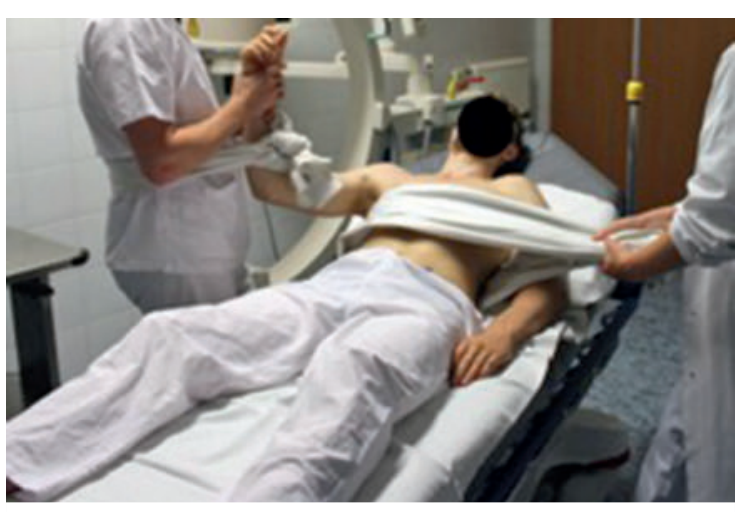

- Abb. 10 Der Helfer kann durch Gegenzug ein Verrutschen des Patienten auf der Unterlage verhindern. Sollte die Schulterreposition trotzdem nicht erfolgreich sein, kann zusätzlich eine Außenrotation der luxierten Schulter versucht werden.

\section{Schulterreposition nach Hippokrates}

Cave

Dieses Repositionsmanöver sollte nur als Notfallvariante betrachtet werden.

Aufgrund des vergleichsweise schmerzhaften und komplikationsträchtigen Ablaufs stehen viele andere Repositionsmanöver im Vordergrund. Beschriebene Verletzungen nach brüsker Reposition nach Hippokrates reichen über Gefäßverletzungen und Plexusschäden bis hin zu Frakturen [5].

\section{Schritt 1 Axialer Zug auf den Arm}

Nach Analgesie bzw. milder Analgosedierung üben Sie axialen Zug auf den Arm aus ( $\bullet$ Abb. 11). 


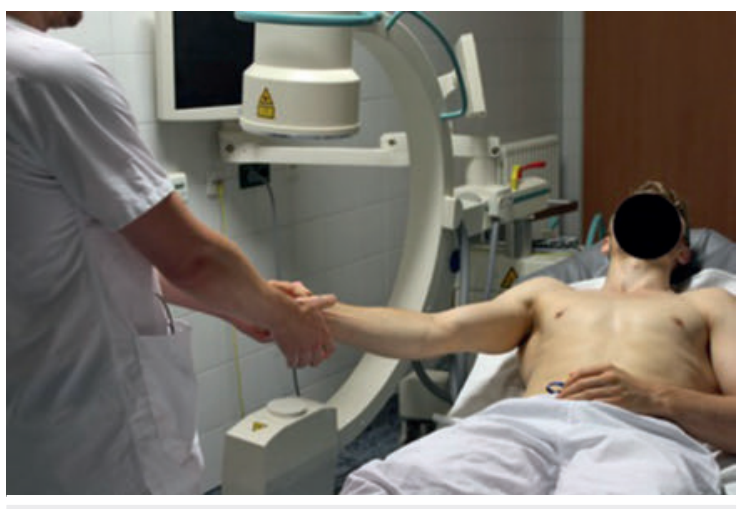

- Abb. 11 Für die Schulterreposition lagern Sie den Patienten in Rückenlage und greifen den Arm proximal des Handgelenks.

\section{Schritt 2 Zug verstärken}

Sie können den Zug auf den Arm verstärken, wenn Sie sich bei der Reposition am Thorax oder in der Achsel des Patienten abstützen ( $\triangleright$ Abb. 12). Ziehen Sie sich das Schuhwerk vor der Reposition aus.

Analog zur Repositionsmethode nach Matsen sollten Sie auf übermäßige Gewalteinwirkung verzichten und, falls nötig, die Analgosedierung vertiefen, um ein Gegenspannen des Patienten zu verhindern.

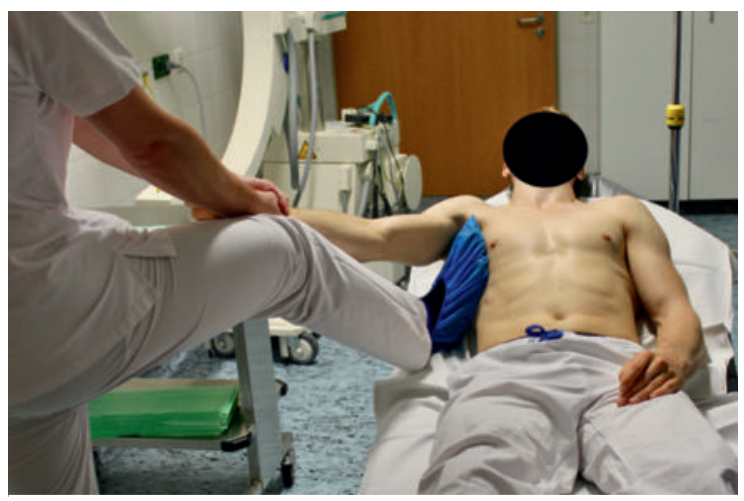

- Abb. 12 Stellen Sie einen Fuß in die Achsel oder gegen den Thorax des Patienten, um bei gleichmäßigem, axialem Zug ein Gegenlager zu erzeugen, bis die Schulter reponiert ist.

\section{Selbstreposition nach „Davos“ \\ (Boss-Holzach-Matter)}

\section{Schritt 1 Handgelenke zusammenbinden}

Für Patienten mit habitueller Schulterluxation oder bei einer Reposition in schwierigem Gelände ohne Hilfsmittel kann die Methode der Selbstreposition genutzt werden. Binden Sie hierfür die Handgelenke des Patienten mit einer Binde locker zusammen ( $\triangleright$ Abb. 13).

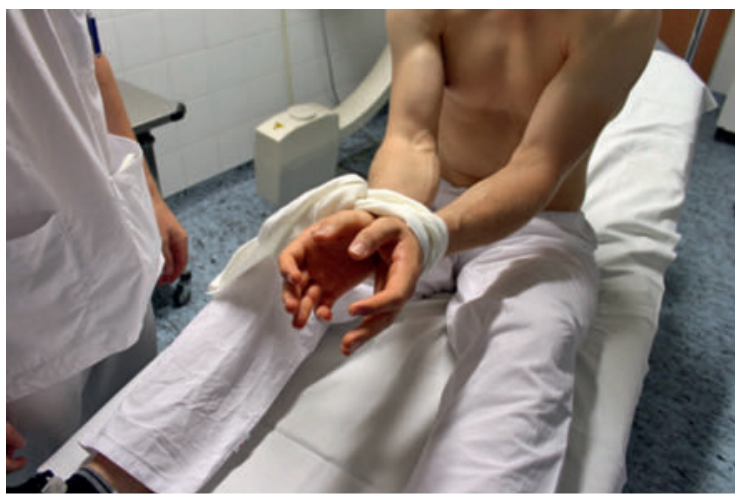

Abb. 13 Binden Sie die Handgelenke des Patienten locker zusammen.

\section{Schritt 2 Handgelenke über das aufgestellte Knie legen}

Applizieren Sie ggf. eine ausreichende Analgesie und lassen Sie den Patienten die zusammengebundenen Handgelenke über das gebeugte Bein der verletzten Seite legen ( $\triangleright$ Abb. 14).

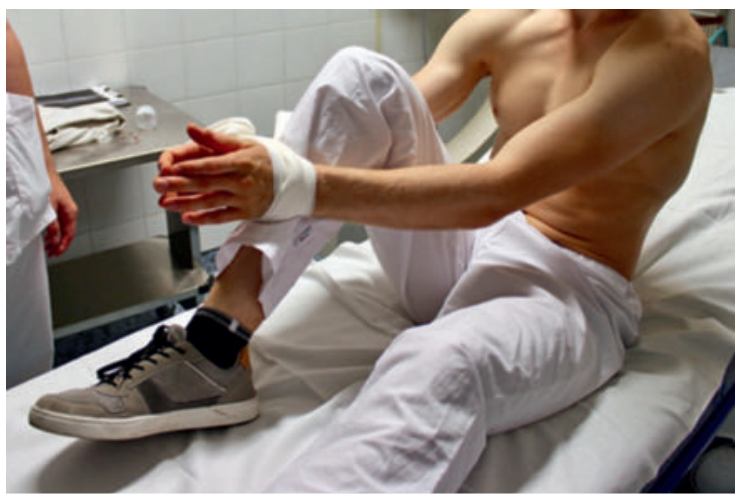

- Abb. 14 Lassen Sie für die Schulterreposition den Patienten seine zusammengebundenen Handgelenke über sein Knie legen. 


\section{Schritt 3 Zug ausüben}

Fordern Sie den Patienten auf, sich zunehmend rückwärts zu lehnen. Dieser kann so selbst die Reposition angepasst an das Schmerzniveau durchführen. Eine schonende Reposition wird somit erreicht. Durch Rückverlagerung des Kopfes kann der Zug auf den Arm langsam gesteigert werden ( $\triangleright$ Abb. 15).

TIPP

Wenn Sie den Fuß des gebeugten Beines fixieren, kann der Patient langsam mehr Zug aufbauen.

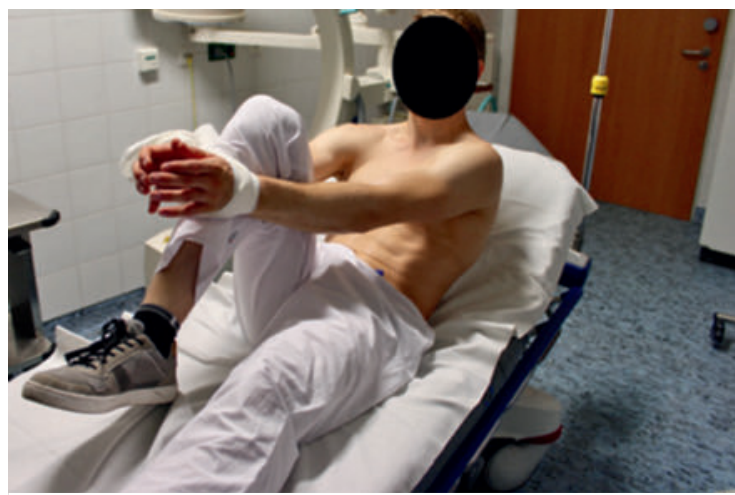

- Abb. 15 Fordern Sie den Patienten auf, sich zurückzulehnen, um so kontinuierlichen Zug auf die Schulter auszuüben, bis die Schulter reponiert ist.

\section{Nach der Reposition}

Nach erfolgreicher Reposition sollte erneut eine Überprüfung und Dokumentation der pDMS erfolgen. Die Ruhigstellung erfolgt mittels einer Bandage in Innenrotation. Ebenfalls obligat ist die erneute Röntgenbildgebung des Schultergelenks in a.-p. und axialem Strahlengang, um die erfolgreiche Reposition zu bestätigen und eventuell aufgetretene knöcherne Läsionen insbesondere am Glenoid auszuschließen.

\section{FAZIT}

Eine Schulterluxation stellt eine gut therapierbare Verletzung dar. Sie kann ganz ohne oder mit einfachen Hilfsmitteln durchgeführt werden. Folgende Punkte sind bei der Schulterreposition zu beachten:

- Eine präklinische Schulterreposition sollte nur bei eingeschränkter pDMS oder nicht absehbarer Transportzeit in die nächste Klinik erfolgen, da ein Frakturausschluss vor Ort schwierig ist.

- Eine ausreichende Analgesie oder Analgosedierung erhöht die Erfolgswahrscheinlichkeit und minimiert das Auftreten von Begleitverletzungen bei der Reposition.

- Brüske Repositionsversuche sind zu vermeiden.

- Bei ausbleibendem Erfolg ist an eine ausreichende Analgesie oder ein Repositionshindernis zu denken.

Interessenkonflikt

Die Autorinnen/Autoren geben an, dass kein Interessenkonflikt besteht.

Autorinnen/Autoren

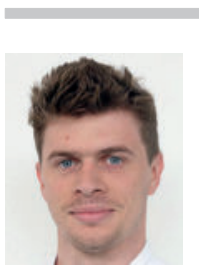

\section{Peter Melcher}

Dr. med. 2010-2016 Studium der Humanmedizin an der Universität Leipzig. Seit 2017 Assistenzarzt an der Klinik für Orthopädie, Unfallchirurgie, Plastische Chirurgie am Universitätsklinikum Leipzig; dort Facharztausbildung zum Facharzt für Orthopädie und Unfallchirurgie. Schwerpunkte: arthroskopische und offene Gelenkchirurgie.

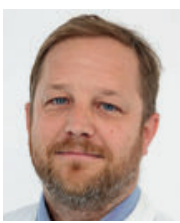

\section{Jan Theopold}

Dr. med. 1998-2005 Studium der Humanmedizin an der Universität Leipzig. 2005-2011 Facharztausbildung und Subspezialisierung, Facharzt für Orthopädie und Unfallchirurgie, Spezielle Unfallchirurgie, Notfallmedizin. Seit 2013 Oberarzt des Bereichs Arthroskopische und spezielle Gelenkchirurgie/Sportverletzungen in der Klinik für Orthopädie, Unfallchirurgie, Plastische Chirurgie am Universitätsklinikum Leipzig. Schwerpunkte: Schulterchirurgie, arthroskopische und offene Gelenkchirurgie. 
Korrespondenzadresse

\section{Dr. med. Peter Melcher}

Universitätsklinikum Leipzig

Haus 4

Liebigstraße 20

04103 Leipzic

peter.melcher@gmx.de

KOORDINATION DER RUBRIK

„SCHRITT FÜR SCHRITT“

Dr. med. Felix Girrbach, Leipzig
Literatur

[1] Cutts S, Prempeh M, Drew S. Anterior shoulder dislocation. Ann R Coll Surg Engl 2009; 91: 2-7

[2] Matsen FA, Zuckerman JD. Anterior glenohumeral instability. Clin Sports Med 1983; 2: 319-338

[3] Minkus M, Böhm E, Moroder P et al. Initiales Management der traumatischen ventralen Schultererstluxation. Unfallchirurg 2018; 121: 100-107

[4] Schliemann B, Schildhauer TA, Seybold D. Die dorsal verhakte Schulterluxation: Diagnostik und Behandlungsstrategien. Obere Extremität 2010; 5: 216-224

[5] Schmal H, Strohm PC, Rosahl SK et al. Verletzung der A. subscapularis durch Reposition einer vorderen Schulterluxation. Unfallchirurg 2006; 109: 153-155

Bibliografie

DOI https://doi.org/10.1055/a-0963-0640

Notfallmedizin up2date 2019; 14: 345-351

(c) Georg Thieme Verlag KG Stuttgart · New York

ISSN 1611-6550 